\title{
Malonyl-proteome profiles of Staphylococcus aureus reveal lysine malonylation modification in enzymes involved in energy metabolism
}

\author{
Yanan Shi ${ }^{1}$, Jingjing Zhu' ${ }^{1}$, Yan Xu' ${ }^{2}$ Xiaozhao Tang${ }^{2}$, Zushun Yang ${ }^{2}$ and Aixiang Huang ${ }^{1 *}$
}

\begin{abstract}
Background: Protein lysine malonylation, a novel post-translational modification (PTM), has been recently linked with energy metabolism in bacteria. Staphylococcus aureus is the third most important foodborne pathogen worldwide. Nonetheless, substrates and biological roles of malonylation are still poorly understood in this pathogen.

Results: Using anti-malonyl-lysine antibody enrichment and high-resolution LC-MS/MS analysis, 440 lysine-malonylated sites were identified in 281 proteins of $S$. aureus strain. The frequency of valine in position -1 and alanine at +2 and + 4 positions was high. KEGG pathway analysis showed that six categories were highly enriched, including ribosome, glycolysis/gluconeogenesis, pentose phosphate pathway (PPP), tricarboxylic acid cycle (TCA), valine, leucine, isoleucine degradation, and aminoacyl-tRNA biosynthesis. In total, 31 malonylated sites in S. aureus shared homology with lysinemalonylated sites previously identified in E. coli, indicating malonylated proteins are highly conserved among bacteria. Key rate-limiting enzymes in central carbon metabolic pathways were also found to be malonylated in S. aureus, namely pyruvate kinase (PYK), 6-phosphofructokinase, phosphoglycerate kinase, dihydrolipoyl dehydrogenase, and F1F0-ATP synthase. Notably, malonylation sites were found at or near protein active sites, including KH domain protein, thioredoxin, alanine dehydrogenase (ALD), dihydrolipoyl dehydrogenase (LpdA), pyruvate oxidase CidC, and catabolite control protein A (CcpA), thus suggesting that lysine malonylation may affect the activity of such enzymes.
\end{abstract}

Conclusions: Data presented herein expand the current knowledge on lysine malonylation in prokaryotes and indicate the potential roles of protein malonylation in bacterial physiology and metabolism.

Keywords: Post-translational modification (PTM), Lysine malonylation, Staphylococcus aureus, Energy metabolism, Enzymes

\section{Background}

The emerging advancements in high-sensitive mass spectrometry and high-quality pan-anti-acyl-lysine antibody have revealed post-translational modifications (PTMs) in the bacterial gene expression, virulence, and protein

\footnotetext{
* Correspondence: yananshihaha@126.com

${ }^{1}$ College of Food Science and Technology, Yunnan Agricultural University, Kunming 650201, Yunnan, China

Full list of author information is available at the end of the article
}

functions $[1,2]$. So far, several PTM types have been identified, i.e., acetylation, crotonylation, succinylation, glutarylation, and 2-hydroxyisobutyrylation. Protein acetylation, a highly-conserved PTM, targets enzymes involved in central carbon metabolism in eukaryotes and various prokaryotes $[3,4]$. Moreover, protein acetylation contributes to secondary metabolism, fatty acid metabolism, protein localization regulation, and synthesis in Thermus thermophilus [5], Salmonella enterica, and certain Gram-positive

C C The Author(s). 2021 Open Access This article is licensed under a Creative Commons Attribution 4.0 International License, which permits use, sharing, adaptation, distribution and reproduction in any medium or format, as long as you give appropriate credit to the original author(s) and the source, provide a link to the Creative Commons licence, and indicate if changes were made. The images or other third party material in this article are included in the article's Creative Commons licence, unless indicated otherwise in a credit line to the material. If material is not included in the article's Creative Commons licence and your intended use is not permitted by statutory regulation or exceeds the permitted use, you will need to obtain permission directly from the copyright holder. To view a copy of this licence, visit http://creativecommons.org/licenses/by/4.0/ The Creative Commons Public Domain Dedication waiver (http://creativecommons.org/publicdomain/zero/1.0/) applies to the data made available in this article, unless otherwise stated in a credit line to the data. 
bacteria, such as Bacillus subtilis and Geobacillus kaustophilus [6, 7]. Zhang et al. first identified 14 succinylated proteins with 69 succinylation sites in Escherichia coli [8]. Subsequently, through the comprehensive analysis of lysine succinylomes in bacteria, succinylation has been characterized to occur in glycolysis, tricarboxylic acid (TCA) cycle, and fatty acid metabolism [9]. Recent studies have described a novel post-translational modification, namely lysine-2-hydroxyisobutyrylation (Khib), on histones of eukaryotic cells with potential involvement in cell transcription and metabolism. Dong et al. found that a lysine de-2-hydroxyisobutyrylase (CobB) is involved in glycolysis regulation by regulating enolase catalytic activities, which further affects bacterial growth [10].

Malonylation, a newly described PTM by means of pan anti-malonylated lysine antibodies and synthetic peptides, involves malonyl-CoA as a cofactor and allows cells to respond to internal and external cues rapidly. Malonylated lysine is negatively charged due to the presence of a carboxylic group, which can impact protein function and regulatory enzymes [11]. Protein acylation modification has been shown to participate in the regulation of enzymatic activity, gene expression, virulence, protein synthesis, translation, and stability, as well as in other biochemical processes. Although malonyl-CoA is considered the most common donor of the malonyl group, malonylation mediating enzymes are still unexplored [11]. SIRT5, a member of the family of lysine deacetylases (KDACs), was found to catalyze lysine demalonylation reaction in mammalian cells [12]. Therefore, it is speculated that both protein acetylation and malonylation are reversibly regulated by lysine acetyltransferases (KATs) and KDACs in mammalian cells. The addition of acetyltransferase inhibitors or histone deacetylases in vitro can significantly inhibit the growth of Trichophyton rubrum by inducing cell apoptosis, or it may point towards a new direction to treat fungal diseases. There has been an increasing interest in exploring the regulatory roles of lysine malonylation (Kmal) in several microbial species, such as Escherichia coli [13], Bacillus amyloliquefaciens [14], and Saccharopolyspora erythraea [15]. However, little is known about the substrates and biological roles of Kmal in S. aureus.

Staphylococcus aureus is a Gram-positive bacterium and the third most common foodborne pathogen worldwide $[16,17]$. Previous studies have shown that raw milk and dairy products are heavily contaminated by $S$. aureus $[18,19]$. Furthermore, S. aureus isolated from several products of animal origin has been shown be to resistant to antibiotics, such as methicillin, vancomycin (glycopeptide), daptomycin (lipopeptide), linezolid (oxazolidinone), tedizolid (anoxazolidinone), dalbavancin (lipoglycopeptide), ceftaroline ( $\beta$-lactam antibiotic) and carbapenems $[20,21]$. Once $S$. aureus contaminates foods, it can multiply under favorable conditions and secrete enterotoxins, which, if ingested, can lead to symptoms of food poisoning, e.g., vomiting. Therefore, S. aureus is an important public health concern.

Therefore, the aim of this study was to characterize malonylated residues in proteins of $S$. aureus using affinity enrichment coupled with mass spectrometry-based techniques. To our knowledge, this study is the first to characterize protein lysine malonylation in S. aureus, and the present findings provide insights into its biological roles in bacterial energy metabolism.

\section{Methods}

\section{Bacterial strain and cell culture}

S. aureus DC.RB_015 was obtained from the Yunnan Center for Disease Control and Prevention, China, and was used throughout the study. S. aureus was inoculated in $100 \mathrm{~mL}$ of Luria Bertani (LB) broth in a five-hundred$\mathrm{mL}$ Erlenmeyer baffled flask under shaking at $150 \mathrm{rpm}$ for $18 \mathrm{~h}$ at $37^{\circ} \mathrm{C}$. Cells at stationary phase $\left(\mathrm{OD}_{600}=0.9\right)$ were harvested by centrifugation at $8000 \times g$ for $5 \mathrm{~min}$ at $4{ }^{\circ} \mathrm{C}$. Before lysis, cells were washed twice with cold PBS.

\section{Protein extraction}

Harvested $S$. aureus cells were frozen by liquid nitrogen and ground into powder, then transferred to a five-mL centrifuge tube. Pellets were resuspended in lysis buffer$8 \mathrm{M}$ Urea in $50 \mathrm{mM} \mathrm{NH}_{4} \mathrm{HCO}_{3}, \mathrm{pH} 8.0$, containing $1 \%$ protease inhibitor cocktail; for PTM experiments, deacetylase inhibitors were also added to lysis buffer, e.g., $3 \mu \mathrm{M}$ trichostatin A (TSA) and $50 \mathrm{mM}$ nicotinamide (NAM) and sonicated for $5 \mathrm{~min}$. After incubation on ice for 30 $\mathrm{min}$, the lysate was sonicated again to disrupt DNA clump. After centrifugation at $12,000 \times g$ at $4{ }^{\circ} \mathrm{C}$ for $10 \mathrm{~min}$, supernatants were transferred to new tubes, and protein was quantified by BCA protein assay kit (Beyotime Biotechnology, China).

\section{Western blotting}

Extracted proteins were standardized to the same concentration $(2.39 \mathrm{mg} / \mathrm{mL})$ and boiled in SDS loading buffer for $10 \mathrm{~min}$. Proteins were then subjected to $12 \%$ SDS-PAGE and transferred to a polyvinylidene difluoride (PVDF) membrane. The membrane was blocked for $2 \mathrm{~h}$ in TBS buffer $(25 \mathrm{mM}$ Tris- $\mathrm{HCl}, \mathrm{pH} 8.0,150 \mathrm{mM} \mathrm{NaCl})$ containing $5 \%$ bovine serum albumin (BSA) with further incubation overnight at $4{ }^{\circ} \mathrm{C}$ with the following: antisuccinyl lysine antibody (catalog no. PTM-419, Lot: 105032317G009, Biolabs Inc., Hangzhou, China) (1:1000, in TBS with $2.5 \%$ BSA); anti-malonyl lysine antibody (catalog no. PTM-902, Lot: 23056103HA07, Biolabs Inc.) (1:1000, in TBS with $2.5 \%$ BSA); anti-acetyl lysine antibody (catalog no. PTM-101, Lot: 10167 J809, Biolabs Inc.) (1:1000, in TBS with $2.5 \% \mathrm{BSA})$; and anti-2- 
hydroxyisobutyryllysine antibody (catalog no. PTM-802, Lot: 13592312JB09, Biolabs Inc.) (1:1000, in TBS with $2.5 \%$ BSA). After three consecutive washes with TBST buffer $(25 \mathrm{mM}$ Tris- $\mathrm{HCl}, \mathrm{pH} 8.0,150 \mathrm{mM} \mathrm{NaCl}, 0.1 \%$ Tween20), the membrane was incubated with goat antimouse IgG $(\mathrm{H}+\mathrm{L})$ antibody horseradish peroxidase conjugate (1:5000; Thermo Fisher Scientific, Waltham, MA, USA) for $1 \mathrm{~h}$ at $37^{\circ} \mathrm{C}$. After washing the membrane three times, an ECL substrate kit was used for protein visualization [14].

\section{Trypsin digestion}

Protein samples were reduced with $5 \mathrm{mM}$ dithiothreitol for $30 \mathrm{~min}$ at $56^{\circ} \mathrm{C}$ and alkylated with $11 \mathrm{mM}$ iodoacetamide for $15 \mathrm{~min}$ at room temperature in darkness. Reduced proteins were diluted by the addition of $100 \mathrm{mM}$ TEAB with urea concentration less than $2 \mathrm{M}$. Trypsin was added at a 1:50 trypsin/protein ratio for first digestion overnight and then at a 1:100 ratio for second fourhour digestion [14]. A total of $12 \mathrm{mg}$ of proteins was used for trypsin digestion.

\section{HPLC fractionation}

Tryptic peptides were fractionated by high $\mathrm{pH}$ reversedphase high performance liquid chromatography (HPLC) as previously described [15] using a BetaSil ${ }^{\text {tw }} \mathrm{C} 18$ column ( $5 \mu \mathrm{m}$ particle size, $10 \mathrm{~mm}$ ID, $250 \mathrm{~mm}$ length; Thermo Scientific). Peptides were separated with an acetonitrile gradient $(2-60 \%)$ in $10 \mathrm{mM}$ ammonium bicarbonate (pH 8.5) for $80 \mathrm{~min}, 0.7 \% / \mathrm{min}$. Peptides were then combined into 10 fractions and vacuum-dried for affinity enrichment.

\section{Affinity enrichment}

Fractionated peptides were dissolved in NETN buffer $(100 \mathrm{mM} \mathrm{NaCl}, 1 \mathrm{mM}$ EDTA, $50 \mathrm{mM}$ Tris- $\mathrm{HCl}, 0.5 \%$ NP-40, pH 8.0) and incubated overnight with $10 \mu \mathrm{L}$ of drained pre-washed antibody beads (Catalog no. PTM904; lot: TAJB09B02; PTM Biolab, China) at $4{ }^{\circ} \mathrm{C}$ under gentle shaking for lysine-malonylated peptide enrichment. Beads were gently washed four times with NETN buffer and twice with double-distilled $\mathrm{H}_{2} \mathrm{O}$. Peptides bound to the beads were eluted with $0.2 \%$ TFA and then vacuum-dried. Eluted peptides were desalted with $\mathrm{C} 18$ tips (Millipore, Billerica, MA, USA), according to manufacturer's instructions [13].

\section{Liquid chromatography-tandem mass spectrometry analysis}

Enriched lysine-malonylated peptides were dissolved in solvent A ( $0.1 \% \mathrm{FA}$ in $2 \% \mathrm{ACN})$ and loaded onto a homemade reversed-phase precolumn $(75 \mu \mathrm{m}$ ID $\times 4 \mathrm{~cm}$ in length, $5 \mu \mathrm{m}$ particle size). The gradient increased from 6 to $23 \%$ solvent B $(0.1 \%$ formic acid in $98 \%$ acetonitrile) during the initial 26 min: from 23 to $35 \%$ during $8 \mathrm{~min}$, climbing to $80 \%$ within $3 \mathrm{~min}$, and then keeping at $80 \%$ for the last $3 \mathrm{~min}$; all gradient changes were performed at a constant flow rate of $400 \mathrm{~nL} / \mathrm{min}$ in an EASY-nLC 1000 UPLC system. Peptides were subjected to a NanoSpray Ionization source followed by tandem mass spectrometry (MS/MS) in a Q Exactive ${ }^{\mathrm{mm}}$ Plus system (Thermo Scientific) coupled online with ultraperformance liquid chromatography using the following parameters: $2.0 \mathrm{kV}$ electrospray voltage, 350 to $1800 \mathrm{~m} / \mathrm{z}$ range for a full scan, and intact peptides were detected in Orbitrap (Thermo Scientific) under a 70,000 resolution. Peptides were then selected for MS/MS using normalized collision energy value set at 28 , and fragments were detected in Orbitrap at a 17,500 resolution. A data-dependent procedure that alternated between one MS scan followed by 20 MS/MS scans with $15.0 \mathrm{~s}$ dynamic exclusion [13].

\section{Database search}

The resulting MS/MS data were processed using the MaxQuant search engine (v.1.5.2.8). Tandem mass spectra were searched against the UniProt Staphylococcus aureus taxonomy database concatenated with a reverse decoy database. Trypsin/P was specified as the cleavage enzyme allowing up to four missing cleavages. Mass tolerance for precursor ions was set at $20 \mathrm{ppm}$ in an initial search and $5 \mathrm{ppm}$ in the main search, and mass tolerance for fragment ions was set at $0.02 \mathrm{Da}$. Carbamidomethylation on cysteine residues was specified as fixed modification, and malonylation and oxidation on methionine residues were specified as variable modifications. False discovery rate (FDR) was adjusted to $<1 \%$, and the minimum score for modified peptides was set $>$ 40 , peptides for quantification: Unique \& razor peptides.

\section{Bioinformatic analysis}

The subcellular localization of modified proteins was predicted by WoLF PSORT (version PSORT/PSORTII) [22]. Predictions of amino acid positions and protein secondary structures were performed using, respectively, motif- $x$ and NetSurfP software [23]. Similarly, nonmalonylated amino acids in protein secondary structures were analyzed with the Swiss-Prot "Staphylococcus aureus" dataset as the negative set. Significance level $(P$ value) was set at 0.05 or lower [24]. Preference of flanking sequences of Kmal sites was detected using iceLogo (version 1.2). In total, 21 peptides containing Kmal sites in the central lysine site and 10 neighboring amino acid residues on both sides ( 21 amino acids in total) were selected as positive sets for analysis. Cytoscape (version 3.3.0) software based on the STRING database (version 11.0) was used to analyze protein-protein interactions of identified malonylated proteins. Interactions that showed 
confidence score higher than or equal to 0.7 from the STRING database were exported to Cytoscape for analysis. Molecular Complex Detection (MCODE) plugin in Cytoscape was used for the analysis of densely connected regions [25]. InterPro (http://www.ebi.ac.uk/ interpro/) and InterProScan software were used to annotate functional domains of all identified proteins. KEGG Automatic Annotation Server (KAAS) was used to obtain the KEGG database description. Annotation was mapped against the KEGG pathway database using the KEGG mapper. InterProScan was used to annotate protein gene ontology (GO) based on sequence homology. A two-tailed Fisher's exact test was applied to GO/ KEGG/Domain enrichment analysis of differentially malonylated proteins against all identified proteins. $P$ values $<0.05$ were considered as statistically significant.

\section{Results and discussion}

Characterization of the lysine-malonylated proteins in $S$. aureus

$S$. aureus cells were collected during the exponential phase, and western blot analysis of whole cell lysates was carried out using pan-anti-acetylation, - 2-hydroxyisobutyrylation, -succinylation, and -malonyl-lysine antibodies. As depicted in Figs. 1 and 2, lysine malonylation is widely spread in the proteome of $S$. aureus. The dot blot analysis was performed to control the specificity of the beads (Fig. S5). Later, global malonylome analysis of S. aureus proteome was performed using affinity enrichment followed by high-resolution LC-MS/MS, as previously reported [26] (Fig. S1)

A total of $440 \mathrm{Kmal}$ sites were identified in 281 proteins of S. aureus (Supplementary Table S1), which were less than the number of malonylated proteins recently identified in E. coli and B. amyloliquefaciens [14], but more than in S. erythraea [15] and Toxoplasma gondii [27]. Among malonylated proteins of $S$. aureus, 191 proteins (67.9\%) contained a single Kmal site, 51 proteins $(18.1 \%)$ contained two Kmal sites, and the remaining had three or more Kmal sites (Fig. S3). Dihydrolipoyl dehydrogenase contained the largest number of malonylated sites $(n=7)$ with a single protein. The second highly malonylated proteins contained six Kmal sites, including phosphoglycerate kinase (PGK) and alanine dehydrogenase (ALD), which are involved, respectively, in the second stage of glycolysis and oxidative deamination of alanine. In the present study, lysine malonylation was found on certain proteins of the $50 \mathrm{~S}$ ribosomal family (L1, L9, L3, L6, L30), and five Kmal sites were also identified in heat shock protein 70 (Hsp70). Previous studies have shown that heat shock proteins are highly succinylated, containing up to 17 independent lysine residues, and are crucial for host immune response regulation during infection by Plasmodium falciparum [28]. The previously reported dihydrolipoyl dehydrogenase had the most intensively acetylated proteins with $15 \mathrm{Kmal}$ sites in Saccharopolyspora erythraea.

GO analysis revealed that malonylated proteins of $S$. aureus were shown to be preferably located in the cytosol (54.7\%) (Supplementary Fig. S4), or as intracellular non-membrane-bounded organelle (11.3\%), ribonucleoprotein complex (14.8\%), cytosolic ribosome (9.6\%), and ribonucleoprotein complex (9.6\%). Several malonylated proteins have been identified in the nucleus, cytoplasm, mitochondria, and chloroplast, thus indicating that a wide variety of biological processes can be potentially regulated by lysine malonylation.

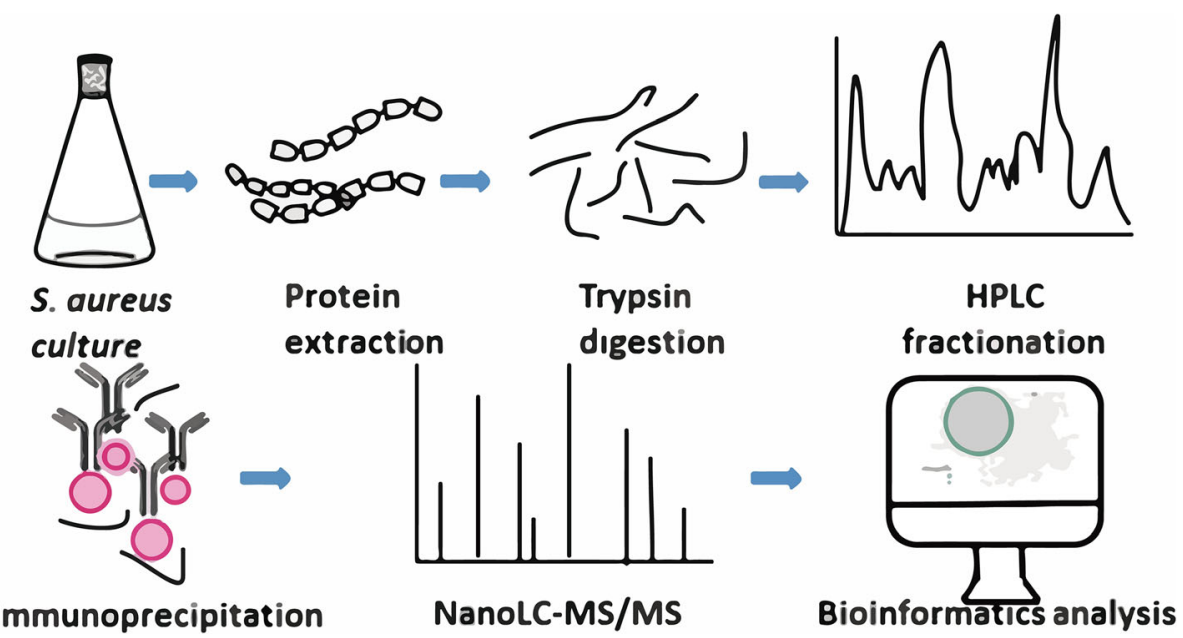

Fig. 1 Workflow for the global profiling of lysine malonylation in S. aureus 


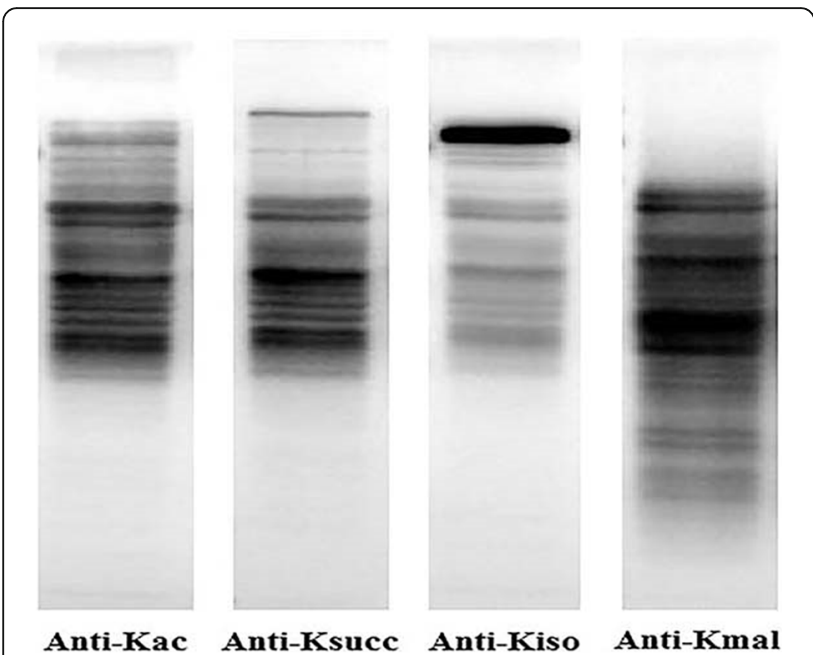

Fig. 2 Western blotting analysis of protein lysine malonylation in Staphylococcus aureus strain DC.RB_015 with anti-acetyl lysine (antiKac), anti-succinyl lysine (anti-Ksucc), anti-2-hydroxyisobutyryl (antiKiso), and anti-malonyl lysine antibodies (anti-Kmal)
Malonylated proteins in S. aureus are involved in central carbon metabolism

A growing body of evidence suggests that lysine malonylation plays a major role in bacterial metabolism regulation. Malonylome analysis of mammalian cells revealed that SIRT5 regulates both cytosolic and mitochondrial proteins with glycolysis as the targeted pathway. KEGG pathway analysis showed that six categories were highly enriched (Fig. 3): ribosome, glycolysis/gluconeogenesis, pentose phosphate pathway (PPP), tricarboxylic acid cycle (TCA), valine, leucine, isoleucine degradation, and aminoacyl-tRNA biosynthesis. Malonylated proteins related to the ribosome pathway were significantly enriched, suggesting a potential involvement of lysine malonylation in protein synthesis. Enrichment of glycolysis/gluconeogenesis, pyruvate metabolism, and citric acid (TCA) cycle KEGG pathways were also observed in E. coli, S. erythraea, Fragaria vesca, and human cells, suggesting that malonylation may control activity or stability of enzymes involved in those pathways and affect the energy metabolism regulation.

Interestingly, nearly all enzymes involved in glycolysis were malonylated at one or more sites (Fig. 4), including critical enzymes 6-phosphofructokinase (PfkA), PGK, and pyruvate kinase (PYK); the latter was found

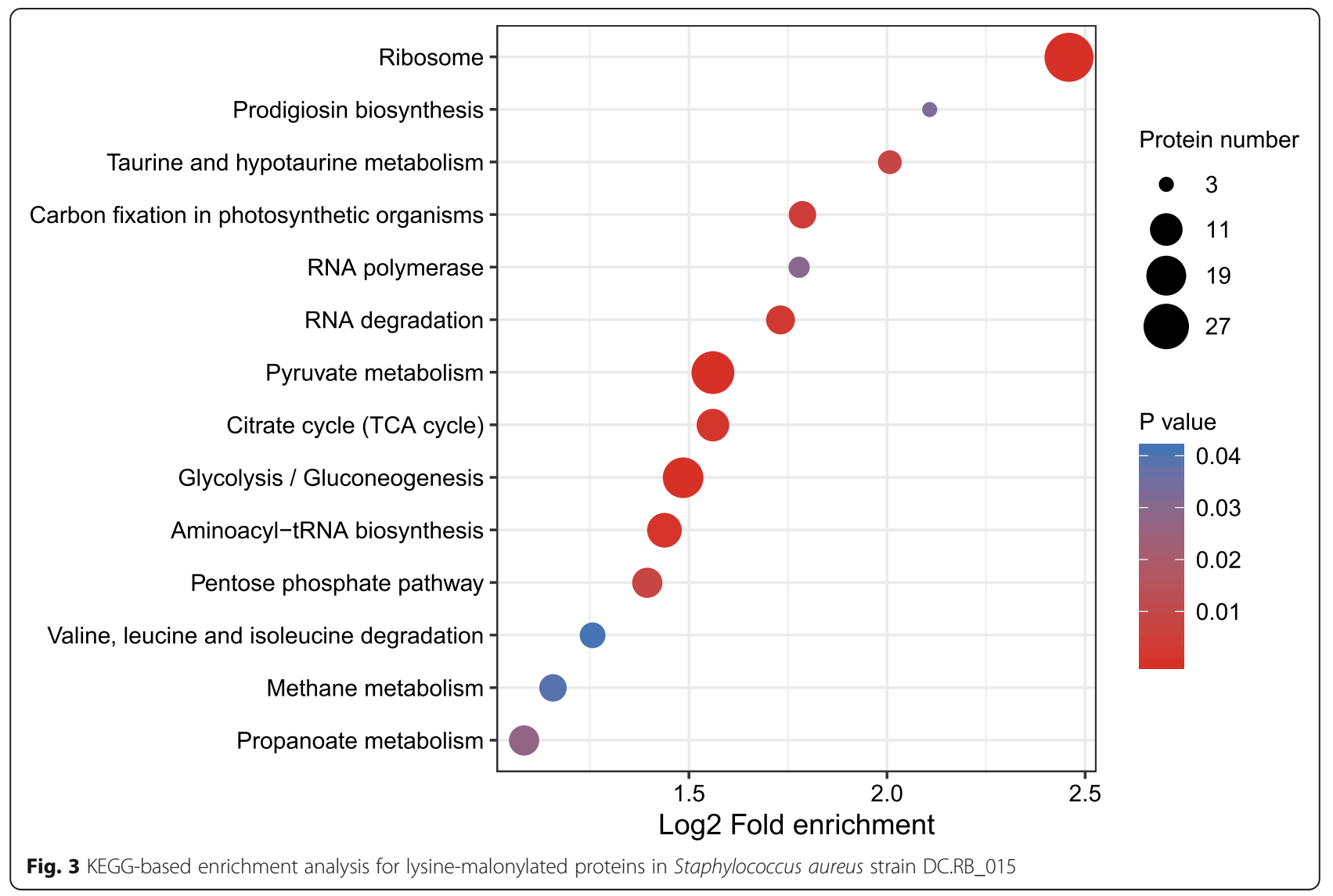




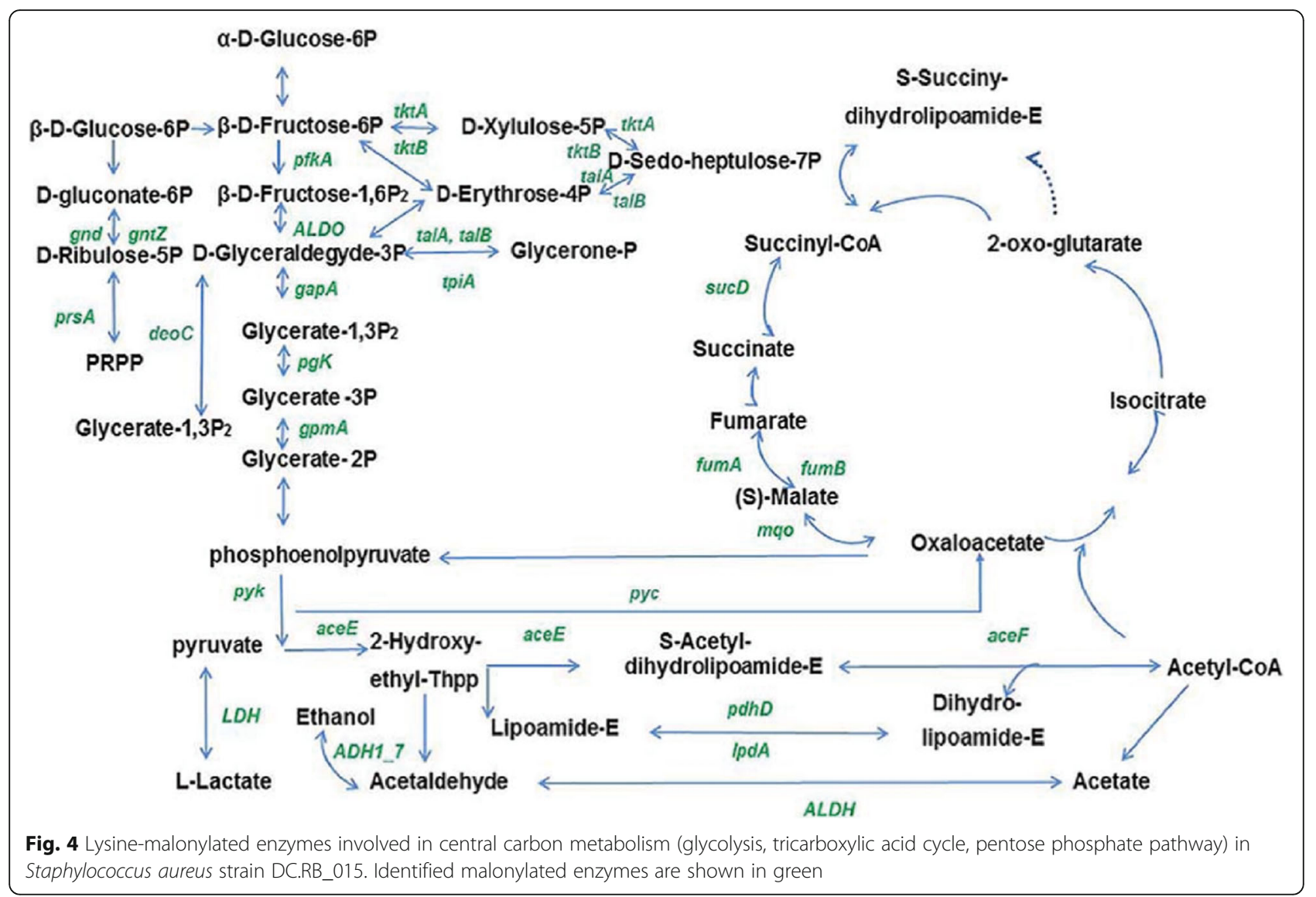

to be malonylated at K66, K433, K434, K435, and K437. Fructose-bisphosphate aldolase plays a central role in glycolysis/gluconeogenesis and may serve as a potential target to fight pathogenic bacteria, and was also found to be malonylated in S. aureus. Moreover, the other six malonylated proteins were found to belong to the pentose phosphate pathway. For instance, LpdA, a subunit of pyruvate dehydrogenase, contained $7 \mathrm{Kmal}$ sites, being also highly malonylated in E. coli (15 Kaml sites). Additionally, three enzymes involved in the TCA cycle, including succinyl-CoA synthetase, fumarate hydratase, and malate dehydrogenase, were also lysinemalonylated in S. aureus. Other malonylated enzymes in $S$. aureus are involved in pyruvate metabolism, namely dihydrolipoyl dehydrogenase (PdhD), LpdA, fructose-bisphosphate aldolase (ALDO) (Fig. 4). Our findings were consistent with previous results in other prokaryotes for which malonylome analysis has been conducted [29]. Considering that the above-mentioned enzymes are mainly related to energy biosynthesis, malonylation is likely to trigger bacterial energy generation.

Acyl-lysine modification can regulate proteinprotein interaction [15]. PPI analysis in the STRING database and PPI networks visualized in Cytoscape helped identify major biological processes affected by
Kmal in S. aureus (Fig. 5). A number of highly associated subnetworks of Kmal proteins were revealed, including glycolysis/gluconeogenesis and ribosomerelated processes, which is consistent with KEGG pathway enrichment analysis.

\section{Enrichment analysis of S. aureus lysine-malonylated proteins}

Enrichment analysis was performed to determine functional categories for lysine-malonylated proteins in $S$. aureus. Most malonylated proteins in S. aureus were significantly related to the following categories: ligase activity, small molecule binding protein, and structural constituent of ribosome (Fig. 6a). Other categories, such as organic cyclic compound binding, heterocyclic compound binding, nucleic acid binding, structural constituent of ribosome, RNA binding, aminoacyl-tRNA synthetases, and elongation factors (FusA, TufA, Efp) were also significantly enriched, suggesting a role of protein malonylation in protein synthesis in S. aureus. Additionally, DnaK and Tig, two proteins involved in protein folding and export, respectively, were also malonylated in S. aureus. DnaK in Salmonella Typhimurium was also found to harbor two Kmal sites (K324, K555) [30]. Synthesized proteins that undergo further modification by 


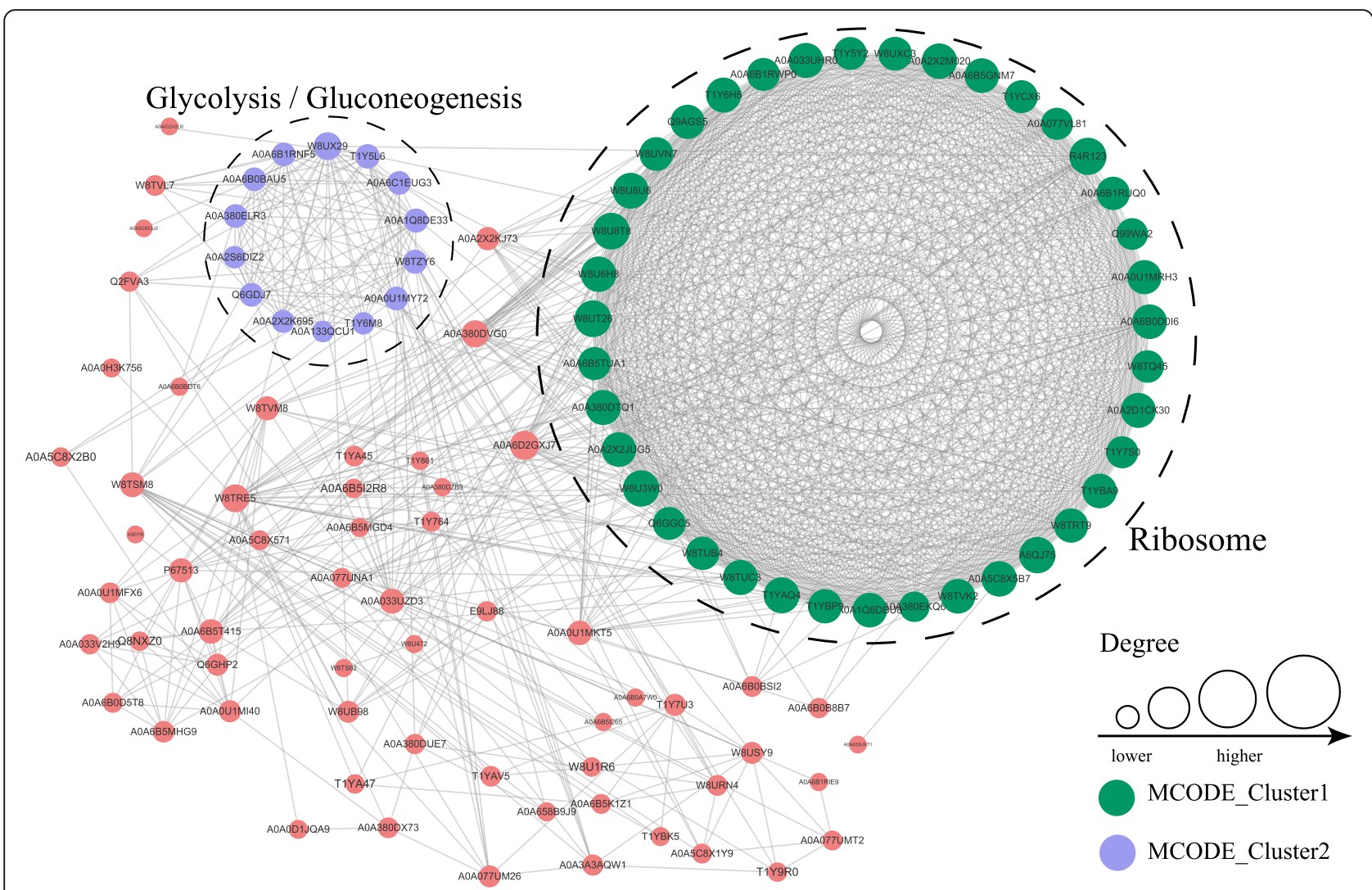

Fig. 5 Protein-protein interaction networks of malonylated proteins in Staphylococcus aureus strain DC.RB_015. Nodes represent malonylated proteins, and edges represent interactors among malonylated proteins. The color of edges indicates the combined score of interactors
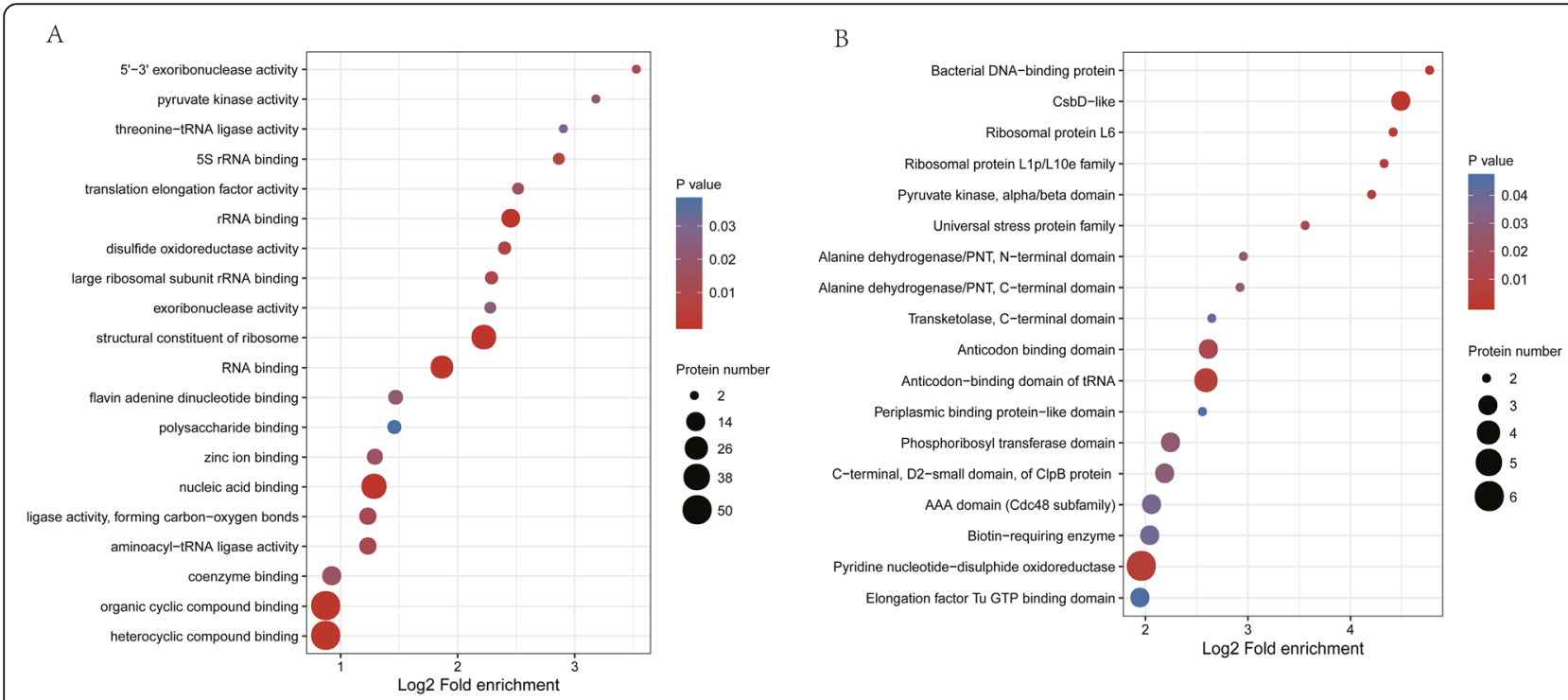

Fig. 6 Gene ontology term enrichment and protein domain annotation of malonylated proteins in Staphylococcus aureus strain DC.RB_015 
chaperones need to find proper localization in the cell by chaperone-mediated transportation. This evidence suggests a potential role of lysine malonylation in controlling protein synthesis in $S$. aureus.

DNA gyrase subunit A (GyrA) in S. aureus was found to be malonylated with one modification site. The malonylated protein fructose-bisphosphate aldolase, which plays an essential role in glycolysis and gluconeogenesis pathways, has been considered a potential target for drug development against pathogenic bacteria [31], were malonylated fructose-bisphosphate aldolase containing 2 Kmal sites in K264 and K265. In addition to the regulation of cell metabolism and protein synthesis, lysine malonylation also modulates gene expression.

In this study, RNA polymerase subunits RpoA, RpoB, and $\mathrm{RpoC}$ were found to contain Kmal sites. RNA polymerase subunits were also found to be highly acetylated in $E$. coli (15 sites in RpoB, 11 in $\mathrm{RpoC}$, and 2 in RpoA) [32]. Moreover, proteins involved in Rho-dependent termination were also lysine-malonylated in S. aureus, suggesting a potential role of lysine malonylation in controlling RNAP promoter specificity, strength, and activity. Additionally, primary free radical scavenging enzymes in $S$. aureus, such as superoxide dismutase, alkylhydroxide peroxidase, thioredoxin, and catalase, also contained malonylated modifications.

The protein domains and functional sites were annotated by InterProScan based on protein sequence alignment using the InterPro database to identify the lysinemalonylated residues in the active protein sites. Among the malonylated peptides in S. aureus, the following signatures and active site motifs were found (Supplementary Table S2): $30 \mathrm{~S}$ ribosomal protein $\mathrm{S} 3$ site in $\mathrm{KH}$ domain protein; thiol reductase thioredoxin site in thioredoxin; alanine dehydrogenase site in alanine dehydrogenase/PNT, N-terminal domain; pyruvate kinase site in pyruvate kinase alpha/beta domain PYK; pyruvate carboxylase site, biotin carboxyl carrier protein of acetylCoA carboxylase site in biotin-requiring enzyme; catabolite control protein site in transcriptional regulator CcpA; pyruvate oxidase site in thiamine diphosphatedependent CidC; dihydrolipoyl dehydrogenase site in pyridine nucleotide-disulphide oxidoreductase LpdA. Several malonylated peptides were also found as signatures in PnpA (an RNA binding domain profile), GlyS (Anticodon binding domain profile), ALD (alanine dehydrogenase/PNT profile), RplA (ribosomal protein L1p/ L10e family) and TufA (elongation factor Tu domain)

A
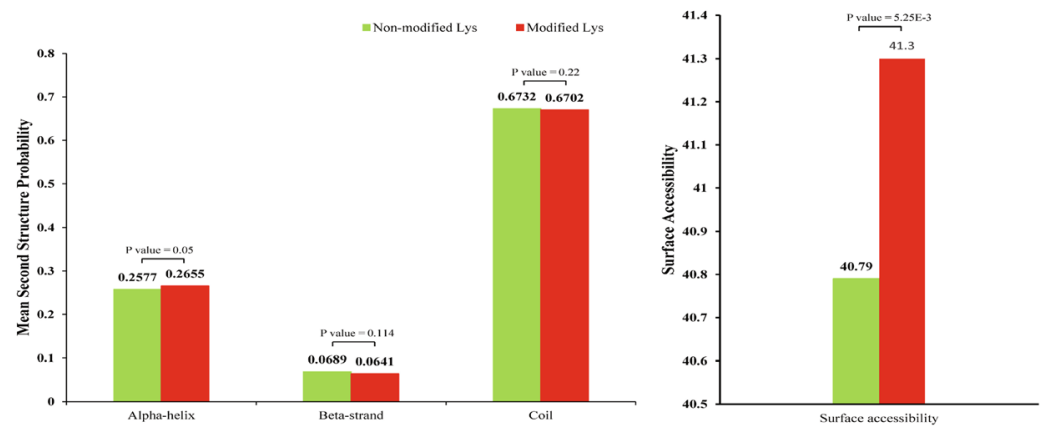

$\mathrm{B}$

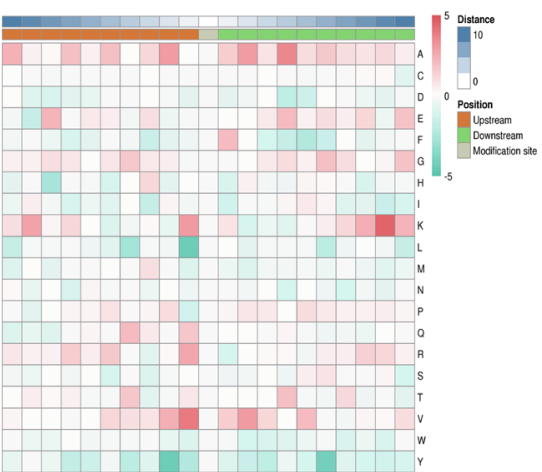

C

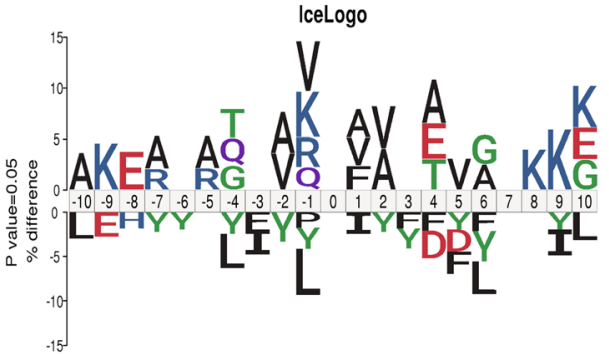

Fig. 7 (A) Occurrence of lysine malonylation in protein secondary structures of Staphylococcus aureus strain DC.RB_015: alpha-helix, beta-strand, and coli. Predicted surface accessibility of malonylation sites. (B) Heat map of amino acids at positions ranging from -10 to +10 around lysinemalonylated residues in S. aureus. Red and green colors indicate high and low frequency, respectively. (C) Sequence analysis of the flanking region of lysine-malonylated sites in S. aureus 
(Fig. 6b). Those findings pinpoint the preferred location of malonyl groups in S. aureus proteins and suggest possible specialized functions of malonylation.

\section{Pattern analysis of malonylated peptides}

Structural analysis of malonylated peptides in S. aureus enabled the identification of surface accessibility of Kmal sites. The average surface accessibility of malonylated lysines was significantly higher $(p<0.005)$ than nonmalonylated lysines (Fig. 7a), which also indicates that Kmal sites are preferably located on the surface of proteins. Certain binding proteins may increase the hydrophobicity of large complexes, thereby interfering with normal assembly in an aqueous environment. Lysine malonylation may thus balance the hydrophobicity by enhancing accessibility to the protein surface. Interestingly, 32.96\% of Kmal sites were located in secondary structure regions; of them, $26.55 \%$ were located in $\alpha$ helices and $6.41 \%$ in $\beta$-sheets regions. The remaining $67.02 \%$ of malonylation sites in S. aureus proteins were located in the unstructured coil regions (Fig. 7a), suggesting that malonylation is more likely to occur in disordered rather than ordered regions in proteins.

Accumulating studies on prokaryotes have proved the preferences for amino-acid residues at particular positions surrounding the acetylated lysine and succinylated lysine [33]. Malonyl-proteins regulated by the same type of enzymes often exhibit similar sequences. Therefore, amino acids around the malonylated lysine from - 10 to +10 were mapped to determine the specific amino acids adjacent to malonylated lysines. As shown in Fig. 7c, the frequency of valine $(\mathrm{V})$ in position -1 and alanine (A) at +2 and +4 positions was high, which is in agreement with heat map analysis (Fig. 7b). Alanine (A) was overly represented around the malonylated lysine in S. aureus, a pattern that is similar to Kmal found in E. coli [13].

\section{Conserved lysine-malonylated proteins in S. aureus and $E$.} coli

Lysine malonylome of $S$. aureus was compared to previously reported $E$. coli malonylomes. Functional classification of $S$. aureus lysine-malonylated proteins is very similar to that of $E$. coli [13]. Interestingly, 31 malonylated sites in S. aureus were considered homologous with E. coli malonylome (Fig. 8; Supplementary Table S3). Most of the acetylated proteins involved in protein synthesis and energy metabolism in $S$. aureus were considered orthologous to $E$. coli. A small subset of lysine-malonylated proteins was commonly found in these two bacterial malonylomes: DNA-binding protein, glyceraldehyde-3-phosphate dehydrogenase, phosphoglycerate kinase, NADP-dependent phosphogluconate dehydrogenase, ATP synthase, 50S ribosomal protein L1, 50S ribosomal protein L3, glyceraldehyde-3-phosphate dehydrogenase, 30S

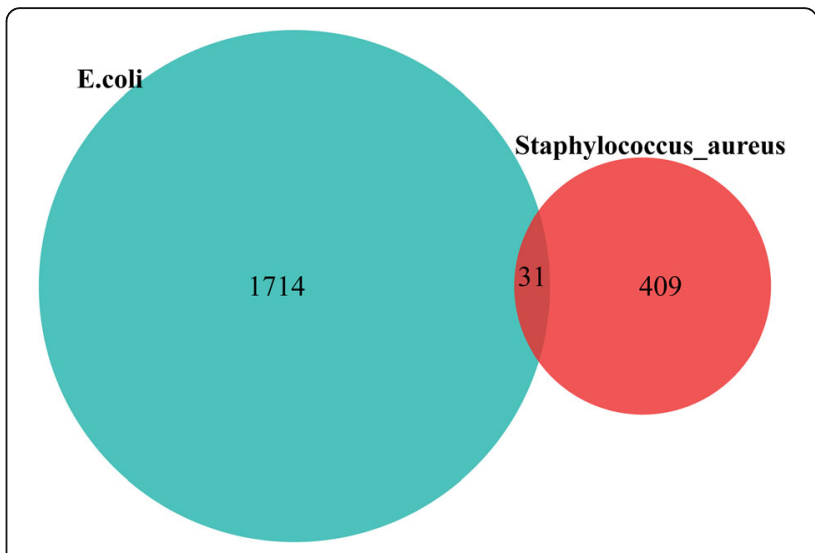

Fig. 8 Venn diagram of identified malonylated sites in previously reported E. coli malonylomes

ribosomal protein S3, dihydrolipoyl dehydrogenase, pyruvate kinase, and formate $\mathrm{C}$-acetyltransferase.

\section{Conclusions}

In the present study, 440 lysine-malonylated sites were described in 281 proteins of $S$. aureus, which confirms the widespread occurrence of lysine malonylation in eubacteria and indicates a conserved PTM shared by eukaryotic and bacterial cells. The findings presented herein expanded the current understanding of lysine malonylation in prokaryotes. More specifically, the malonylated sites were described to occur in key metabolic enzymes involved in several important cellular pathways in S. aureus, such as glycolysis/gluconeogenesis, pyruvate metabolism, TCA cycle, and protein synthesis. Moreover, malonylated sites were found at or near active sites of several proteins, indicating the important role of Kmal in the functional regulation of essential proteins in $S$. aureus. Finally, this study provides useful resources for further functional investigations of lysine malonylation in bacteria.

\section{Supplementary Information}

The online version contains supplementary material available at https://doi. org/10.1186/s12953-020-00169-1.

Additional file 1: Fig. S1. Identification of malonylated peptides by mass tolerance distribution.

Additional file 2: Fig. S2. Identification of malonylated peptides by length.

Additional file 3: Fig. S3. Distribution of malonylated proteins based on the number of malonylated peptides.

Additional file 4: Fig. S4. Cellular component ontology of identified malonylated proteins.

Additional file 5: Fig. S5. Dot blot experiment.

Additional file 6: Table S1. Annotated MS/MS spectra of malonylated peptides and sites in Staphylococcus aureus. 
Additional file 7: Table S2. Domain enrichment analysis of proteins corresponding to modification sites in Staphylococcus aureus.

Additional file 8: Table S3. Conservative analysis of the identified malonylated sites in Staphylococcus aureus and previously reported E. coli malonylomes.

Additional file $\mathbf{9}$ Table S4. Standardization of protein concentration.

\section{Acknowledgments}

We would like to extend our heartful gratitude to Jingjie PTM Biolabs (Hangzhou, China) for assistance in LC-MS/MS analysis. We also thank Ren Xiang for his kind help in strain identification and preservation of Staphylococcus aureus strains, which provides support to the Yunnan Center for Disease Control and Prevention.

\section{Authors' contributions}

Study design: AXH, YX, ZSY. Data collection: YNS, JJZ, and XZT. Methodology: YNS. Data analysis: YNS and AXH. Data interpretation, prepared Figures and Tables: YNS and JJZ. Project administration: YX and ZSY; Resources: JJZ and XZT; Writing-original draft: YNS; Writing-review \& editing: AXH, YNS. The authors read and approved the final manuscript.

\section{Funding}

This study was supported by the National Natural Science Foundation of China (Grant No. 32060572), the Key Yunnan Agricultural Foundation Project (Grant No. 2018FG001-011), and the Key Research and Development Plan of Yunnan Province (Grant No. 2018 BC006-2).

\section{Availability of data and materials}

The datasets used and analyzed during the current study are available from the corresponding authors on reasonable request.

\section{Ethics approval and consent to participate}

Not applicable.

\section{Consent for publication}

All the authors have read and approved the manuscript for publication.

\section{Competing interests}

The authors declare that they have no competing interests.

\section{Author details}

${ }^{1}$ College of Food Science and Technology, Yunnan Agricultural University, Kunming 650201, Yunnan, China. ${ }^{2}$ Yunnan Center for Disease Control and Prevention, Kunming 650201, Yunnan, China.

Received: 19 October 2020 Accepted: 16 December 2020 Published online: 12 January 2021

\section{References}

1. Cain JA, Solis N, Cordwell SJ. Beyond gene expression: the impact of protein post-translational modifications in bacteria. J Proteome. 2014;97:265-86.

2. Rogers LD, Overall CM. Proteolytic post-translational modification of proteins: proteomic tools and methodology. Mol Cell Proteomics. 2013;113: 3532-42.

3. Hirschey MD, Zhao Y. Metabolic regulation by lysine malonylation, succinylation and glutarylation. Mol Cell Proteomics. 2015:1-22.

4. Zhao S, Xu W, Jiang W, Yu W, Lin Y, Zhang T, Yao J, Zhou L, Zeng Y, Li H, et al. Regulation of cellular metabolism by protein lysine acetylation. Science. 2010;327:1000-4.

5. Okanishi H, Kim K, Masui R, Kuramitsu S. Acetylome with structural mapping reveals the significance of lysine acetylation in Thermus thermophilus. J Proteome Res. 2013;12:3952-68.

6. Kim D, Yu BJ, Kim JA, Lee YJ, Choi SG, Kang S, Pan JG. The acetylproteome of gram-positive model bacterium Bacillus subtilis. Proteomics. 2013;13: 1726-36.

7. Lee DW, Kim D, Lee YJ, Kim JA, Choi JY, Kang S, Pan JG. Proteomic analysis of acetylation in thermophilic Geobacillus kaustophilus. Proteomics. 2013;13: 2278-82.
8. Zhang Z, Tan M, Xie Z, Dai L, Chen Y, Zhao Y. Identification of lysine succinylation as a new post-translational modification. Nat Chem Biol. 2011; 7:58-63.

9. Weinert BT, Scholz C, Wagner SA, lesmantavicius V, Su D, Daniel JA Choudhary $C$. Lysine succinylation is a frequently occurring modification in prokaryotes and eukaryotes and extensively overlaps with acetylation. Cell Rep. 2013;4:842-51.

10. Dong H, Zhai G, Chen C, Bai X, Tian S, Hu D, Fan E, Zhang K. Protein lysine de-2-hydroxyisobutyrylation by CobB in prokaryotes. Sci Adv. 2019;5:1-13.

11. Peng C, Lu Z, Xie Z, Cheng Z, Chen Y, Tan M, Luo H, Zhang Y, He W, Yang K, Zhao Y: The first identification of lysine Malonylation substrates and its regulatory enzyme. Mol Cell Proteomics 2011, 111:1-12.

12. Nishida Y, Rardin MJ, Carrico C, He W, Sahu AK, Gut P, Najjar R, Fitch M, Hellerstein M, Gibson BW, Verdin E. SIRT5 regulates both cytosolic and mitochondrial protein Malonylation with glycolysis as a major target. Mol Cell. 2015;59:321-32

13. Qian L, Nie L, Chen M, Liu P, Zhu J, Zhai L, Tao SC, Cheng Z, Zhao Y, Tan M. Global profiling of protein lysine Malonylation in Escherichia coli reveals its role in energy metabolism. J Proteome Res. 2016;15:2060-71.

14. Fan B, Li YL, Li L, Peng XJ, Bu C, Wu XQ, Borriss R. Malonylome analysis of rhizobacterium Bacillus amyloliquefaciens FZB42 reveals involvement of lysine malonylation in polyketide synthesis and plant-bacteria interactions. J Proteome. 2017;154:1-12.

15. $X u$ J-Y, Xu Z, Zhou Y, Ye B-C. Lysine Malonylome may affect the central metabolism and erythromycin biosynthesis pathway in Saccharopolyspora erythraea. J Proteome Res. 2016;15:1685-701.

16. Sergelidis D, Angelidis AS. Methicillin-resistant Staphylococcus aureus: a controversial food-borne pathogen. Lett Appl Microbiol. 2017;64:409-18.

17. Wu S, Duan N, Gu H, Hao L, Ye H, Gong W, Wang Z. A review of the methods for detection of Staphylococcus aureus enterotoxins. Toxins (Basel). 2016;8:1-20.

18. Kümmel J, Stessl B, Gonano M, Walcher G, Bereuter O, Fricker M, Grunert T, Wagner M, Ehling-Schulz M. Staphylococcus aureus entrance into the dairy chain: Tracking S. aureus from dairy cow to cheese. Front Microbiol. 2016:7:1-11.

19. Xing X, Zhang Y, Wu Q, Wang X, Ge W, Wu C. Prevalence and characterization of Staphylococcus aureus isolated from goat milk powder processing plants. Food Control. 2016;59:644-50.

20. Chan LC, Basuino L, Diep B, Hamilton S, Chatterjee SS, Chambers HF. Ceftobiprole- and ceftaroline-resistant methicillin-resistant Staphylococcus aureus. Antimicrob Agents Ch. 2015;59:2960-3.

21. Rodvold KA, McConeghy KW. Methicillin-resistant Staphylococcus aureus therapy: past, present, and future. Clin Infect Dis. 2014; 58(Suppl 1):S20-7.

22. Horton P, Park KJ, Obayashi T, Fujita N, Harada H, Adams-Collier CJ, Nakai K. WoLF PSORT: protein localization predictor. Nucleic Acids Res. 2007;35: W585-7.

23. Xie L, Liu W, Li Q, Chen S, Xu M, Huang Q, Zeng J, Zhou M, Xie J. First succinyl-proteome profiling of extensively drug-resistant mycobacterium tuberculosis revealed involvement of succinylation in cellular physiology. Proteome Res. 2015;14:107-19.

24. Colaert N, Helsens K, Martens L, Vandekerckhove J, Gevaert K. Improved visualization of protein consensus sequences by iceLogo. Nat Methods. 2009:6:786-7.

25. Shannon P, Ramage D, Markiel A, Ozier O, Baliga NS, Wang JT, Ideker T. Cytoscape: a software environment for integrated models of biomolecular interaction networks. Genome Res. 2019:2498-504.

26. Colak G, Pougovkina O, Dai L, Tan M, Brinke H, Huang H, Cheng Z, Park J, Wan $X$, Liu X, et al. Proteomic and biochemical studies of lysine malonylation suggests its malonic aciduria-associated regulatory role in mitochondrial function and fatty acid oxidation. Mol Cell Proteomics. 2015;14:3056-71.

27. Nie LB, Liang QL, Du R, Elsheikha HM, Han NJ, Li FC, Zhu XQ. Global proteomic analysis of lysine Malonylation in toxoplasma gondii. Front Microbiol. 2020;11:1-11.

28. Li X, Hu X, Wan Y, Xie G, Li X, Chen D, Cheng Z, Yi X, Liang S, Tan F. Systematic identification of the lysine succinylation in the protozoan parasite toxoplasma gondii. J Proteome Res. 2014;13:6087-95.

29. Wang Q, Zhang Y, Yang C, Xiong H, Lin Y, Yao J, Li H, Xie L, Zhao W, Yao Y, et al. Acetylation of metabolic enzymes coordinates carbon source utilization and metabolic flux. Science. 2010;327:1004-7.

30. Takaya A, Tomoyasu T, Matsui H, Yamamoto T. The DnaK/DnaJ chaperone machinery of Salmonella enterica serovar Typhimurium is essential for 
invasion of epithelial cells and survival within macrophages, leading to systemic infection. Infect Immun. 2004;72:1364-73.

31. Ziveri J, Tros F, Guerrera IC, Chhuon C, Audry M, Dupuis M, Barel M, Korniotis S, Fillatreau S, Gales L, et al. The metabolic enzyme fructose-1,6bisphosphate aldolase acts as a transcriptional regulator in pathogenic Francisella. Nat Commun. 2017:8:1-15.

32. Lima BP, Antelmann H, Gronau K, Chi BK, Becher D, Brinsmade SR, Wolfe AJ. Involvement of protein acetylation in glucose-induced transcription of a stress-responsive promoter. Mol Microbiol. 2011;81:1190-204.

33. Liu L, Wang G, Song L, Lv B, Liang W. Acetylome analysis reveals the involvement of lysine acetylation in biosynthesis of antibiotics in Bacillus amyloliquefaciens. Sci Rep. 2016;6:1-11.

\section{Publisher's Note}

Springer Nature remains neutral with regard to jurisdictional claims in published maps and institutional affiliations.

Ready to submit your research? Choose BMC and benefit from:

- fast, convenient online submission

- thorough peer review by experienced researchers in your field

- rapid publication on acceptance

- support for research data, including large and complex data types

- gold Open Access which fosters wider collaboration and increased citations

- maximum visibility for your research: over $100 \mathrm{M}$ website views per year

At BMC, research is always in progress.

Learn more biomedcentral.com/submissions 